\title{
Detection of Aspergillus fumigatus mycotoxins: immunogen synthesis and immunoassay development
}

\author{
M. Fox, G. Gray, K. Kavanagh, C. Lewis, S. Doyle* \\ National Institute for Cellular Biotechnology, Department of Biology, National University of Ireland Maynooth, Maynooth, Co. Kildare, Ireland
}

Received 25 August 2003; received in revised form 21 October 2003; accepted 23 October 2003

\begin{abstract}
Immunological detection of secreted low molecular weight toxins represents a potentially novel means of diagnosing infection by the fungus Aspergillus fumigatus. Two such metabolites, gliotoxin and helvolic acid, were selected and conjugated to thyroglobulin for antisera generation in rabbits. Gliotoxin was initially activated using $N$-[ $p$-maleimidophenyl] isocyanate (PMPI) and subsequently conjugated to $S$-acetyl thioglycolic acid $N$-hydroxysuccinimide-activated thyroglobulin, whereas helvolic acid was activated with $N$-(3-Dimethylaminopropyl)- $N^{\prime}$-ethylcarbodiimide (EDC) in the presence of thyroglobulin prior to immunisation. To facilitate subsequent antisera evaluation, both toxins were similarly conjugated to bovine serum albumin (BSA). Matrix-Assisted Laser Desorption Ionisation-Time Of Flight (MALDI-TOF) mass spectrometry and SDSPAGE analysis confirmed covalent attachment of toxins to BSA in the ratios of 15 and $2.4 \mathrm{~mol}$ per mol BSA for gliotoxin and helvolic acid, respectively. Resultant high titer antisera were capable of detecting both BSA-conjugated toxins (inhibitory concentration (IC) $)_{50}: 4-5 \mu \mathrm{g} / \mathrm{ml}$ ). Free toxins were also detectable by competitive immunoassay, whereby $10 \mu \mathrm{g} / \mathrm{ml}$ free gliotoxin $(30 \mu \mathrm{M})$ and helvolic acid $(17 \mu \mathrm{M})$, respectively, inhibited antibody binding to cognate toxin-BSA previously immobilised on microwells. This work confirms that sensitive and specific antisera can be raised against fungal toxins and may have an application in diagnosing fungal infection.
\end{abstract}

(C) 2003 Elsevier B.V. All rights reserved.

Keywords: Gliotoxin; ELISA; MALDI-TOF; Hapten; Immunocompromised

\section{Introduction}

The filamentous fungus Aspergillus fumigatus is responsible for a range of pulmonary infections in immunocompromised patients and those with preexisting lung damage (Fraser, 1993; Daly and Kavanagh, 2001). Invasive aspergillosis is the most

* Corresponding author. Tel.: +353-1-7083858; fax: +353-17083845.

E-mail address: sean.doyle@may.ie (S. Doyle). serious form of aspergillosis, has a mortality rate of $80-95 \%$ and occurs almost exclusively in individuals with pre-existing lung damage or disease and in those immunocompromised as a result of disease or therapy (Denning, 1998). In this form of the disease, there is invasion and necrosis of the lung wall, in addition to whole body fungal dissemination, which results in the infection of a wide range of organs (Daly and Kavanagh, 2001). Despite aggressive anti-fungal chemotherapy, death usually results 7-14 days post-diagnosis (Denning, 1996). As part of its complement of virulence attributes $A$. 
fumigatus produces a range of toxins, most predominantly the immunosuppressive gliotoxin (Amitani et al., 1995a,b), and enzymes (proteases, elastases, phospholipases) which hinder the host immune response and facilitate tissue penetration, respectively (Rinaldi, 1983). Furthermore, extracts obtained from aspergillosis patient sputum have been shown to damage human respiratory epithelial cells (Amitani et al., 1995a). Subsequent analysis confirmed that gliotoxin derived from clinical isolates of A. fumigatus was the toxic agent and that helvolic acid also caused complete ciliostasis and epithelial cell disruption (Amitani et al., 1995b).

Current immunological tests to assess the presence of aspergillosis primarily rely upon the detection of Aspergillus antigens associated with the fungal cell wall (mannans or galactomannans), however, suboptimal sensitivity and specificity have resulted in limited clinical application (Meunier, 1996; Richardson and Kokki, 1998). Weig et al. (2001a,b) have proposed the use of anti-mitogillin (Aspf1) antibody as an alternative method for the detection of aspergillosis and, although controversial (Woo et al., 2001), this method may prove to have a useful clinical application. More recently, Woo et al. (2002) have developed ELISA systems which detect $A$. fumigatus galactomannan (Afmp1p) and anti-Afmplp antibody in invasive aspergillosis patients resulting in a combined sensitivity of $86.7 \%$. Although the application of Aspergillus DNA detection systems has proven useful in terms of correlation in fungal DNA reduction with disease resolution and treatment efficacy, the inability of nucleic acid-based systems to differentiate between (i) fungal strains and (ii) colonisation and infection remains problematical (Yeo and Wong, 2002).

Obviously, there is significant under-detection of aspergillosis and in an effort to overcome this problem, novel methods for the detection of gliotoxin (e.g., RP-HPLC and in vitro cell-based systems which mimic the interaction of fungal hyphae with human tissue) have been developed (Belkacemi et al., 1999; Daly and Kavanagh, 2002). Although these systems have excellent potential for confirmation of Aspergillus infection they are not amenable to routine use in diagnostic laboratories. Detection of specific fungal metabolites has been discussed as an alternative to antibody, antigen or nucleic acid-based tests (Yeo and Wong, 2002). Gliotoxin, a well-characterised fungal metabolite, has potent immunosuppressive effects and is indicative of invasive aspergillosis (Denning, 1998). Similarly, Mitchell et al. (1997) have shown that helvolic acid, produced by A. fumigatus, inhibits the oxidative burst of macrophages. Consequently, the appearance of these toxins may be indicative of invasive aspergillosis since neither toxin has been associated with infections caused by other clinically relevant fungi. Thus, development of immunoassays, which could detect one or more toxins, produced by the genus Aspergillus, would represent a more reliable means of identifying underlying fungal infection and facilitate an effective treatment protocol. The aims of the work presented here were to determine if antibodies could be produced against fungal metabolites and to assess the functionality of any resultant antibodies by enzyme immunoassay.

\section{Materials and methods}

\subsection{Chemicals}

All materials were purchased from SigmaAldrich Chemical (Dorset, UK) unless otherwise stated.

\subsection{Preparation of helvolic acid-protein conjugates}

Helvolic acid was prepared at $1 \mathrm{mg} / \mathrm{ml}$ in dimethyl sulfoxide (DMSO). Briefly, $100 \mu \mathrm{l} N$-(3-Dimethylaminopropyl)- $N^{\prime}$-ethylcarbodiimide (EDC; $10 \mathrm{mg} / \mathrm{ml}$ in deionised water) was added to $25 \mu \mathrm{l}$ of the toxin to facilitate carboxyl group activation (Fig. 1), representing a 100-fold molar excess of EDC over toxin. Immediately, $100 \mu \mathrm{l}$ of either bovine serum albumin (BSA) or thyroglobulin $(10 \mathrm{mg} / \mathrm{ml}$ in $100 \mathrm{mM}$ Methanesulphonic acid, $500 \mathrm{mM} \mathrm{NaCl} \mathrm{pH}$ 6.0) was added to the helvolic acid/EDC mixture, agitated gently and the reaction allowed to proceed for $2 \mathrm{~h}$ at room temperature. This combination of reactants represented a 3- and 0.3-fold molar ratio of activated helvolic acid to BSA and thyroglobulin, respectively. Addition of hydroxylamine $(10 \mathrm{mM}$ final concentration) terminated the reaction. The resultant conjugates 
<smiles>CN1C(=O)C23CC4=CC=CC(O)C4N2C(=O)C1(CO)SS3</smiles>

Gliotoxin<smiles>CC(=O)OC1CC2(C)C(CCC3C(OC(C)=O)C(=O)C4C(C)C(=O)C=CC4(C)C3C2(C)C)/C1=C(\CCC=C(C)C)C(=O)O</smiles>

Helvolic acid

Fig. 1. Structures of gliotoxin and helvolic acid used for conjugation to thyroglobulin and bovine serum albumin (BSA), respectively. Gliotoxin contains two hydroxyl groups available for activation using $N$-[ $p$-maleimidophenyl] isocyanate (PMPI). Helvolic acid contains a single carboxyl group which can be activated using $N$-(3Dimethylaminopropyl)- $N^{\prime}$-ethylcarbodiimide (EDC) prior to protein conjugation.

were dialysed twice, for $4 \mathrm{~h}$ on each occasion with stirring, at $4{ }^{\circ} \mathrm{C}$ against either phosphate buffered saline (PBS) (prior to immunisation and subsequent ELISA analysis) or deionised $\mathrm{H}_{2} \mathrm{O}$ prior to mass spectrometry.

\subsection{Preparation of gliotoxin-BSA conjugates}

$S$-acetyl thioglycolic acid $N$-hydroxysuccinimide (SATA; $1 \mathrm{mg} / 50 \mu \mathrm{l}$ dimethylformamide (DMF)) was added to $10 \mathrm{ml}$ of BSA $(1 \mathrm{mg} / \mathrm{ml}$ in $50 \mathrm{mM}$ potassium phosphate, $150 \mathrm{mM} \mathrm{NaCl}, 1 \mathrm{mM}$ EDTA (Buffer A), pH 7.8). The solution was mixed gently, allowed to incubate for $1 \mathrm{~h}$ and dialysed extensively against Buffer A at pH 6.8. Thyroglobulin was similarly activated with SATA. The extent of SATA incorporation was measured, and reactive sulphydral groups exposed (deblocked), as previously described (Duncan et al., 1983). Gliotoxin (2 $\mathrm{mg} / \mathrm{ml}$ in DMSO) was activated through available hydroxyl groups (Fig. 1) by adding 50 to $105 \mu \mathrm{N}$ [p-maleimidophenyl] isocyanate (PMPI; $30 \mathrm{mg} / \mathrm{ml}$ in DMSO) (Annunziato et al., 1993) and brought to a final volume of $310 \mu \mathrm{l}$ with DMSO which represented a 5-fold molar excess of PMPI over gliotoxin. After reacting for $1 \mathrm{~h}$ at room temperature, PMPI-activated gliotoxin $(260 \mu \mathrm{l})$ was added to $2 \mathrm{ml}$ deblocked SATA-BSA or SATA-thyroglobulin $(0.5 \mathrm{mg} / \mathrm{ml}$ in Buffer A pH 6.8). This combination of reactants represented a 20- and 2-fold molar excess of activated gliotoxin to BSA and thyroglobulin, respectively. After $2 \mathrm{~h}$ incubation, conjugates were dialysed as described above (Section 2.2). Both toxin-protein conjugates were analysed by SDS-PAGE, Western blotting and MatrixAssisted Laser Desorption Ionisation-Time Of Flight (MALDI-TOF) mass spectrometry.

\subsection{Polyclonal antibody generation}

Four rabbits (New Zealand White female rabbits. Age range: 6-12 months. Weight range: $3-4 \mathrm{~kg}$. Obtained from Harlan UK, Oxon, UK) were immunised in total, two with each toxin-thyroglobulin conjugate. Animals were initially immunised subcutaneously with $50 \mu \mathrm{g}$ of each thyroglobulin-toxin conjugate in Freunds complete adjuvant (final volume $=3 \mathrm{ml}$ (50:50 Freunds complete adjuvant: conjugate in PBS)). For subsequent immunisations (four, at two-weekly intervals) $50 \mu \mathrm{g}$ of each thyroglobulintoxin conjugate in Freunds incomplete adjuvant (50:50 with conjugate in PBS) was used until a satisfactory titer (antigen detection $(1 \mu \mathrm{g})$ by Western blot at greater than or equal to $1 / 2000$ antisera dilution) was achieved. Immunisations were carried out according to Irish Department of Health Licence B100/2622.

\subsection{Protein-toxin conjugate analysis}

Free and modified toxin-BSA conjugates were evaluated by SDS-PAGE and Western blot analysis (Ennis et al., 2001). Briefly, electrotransferred proteins were detected using diluted immune antiserum 
A.

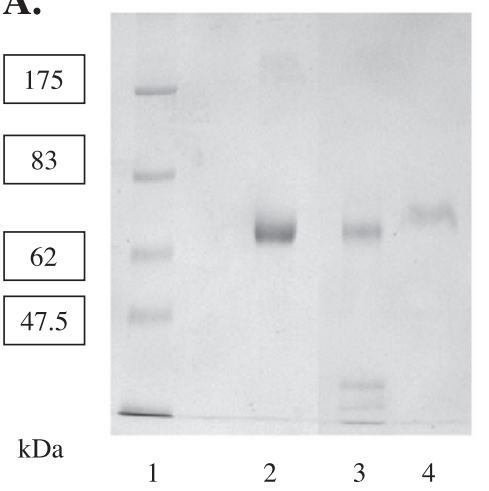

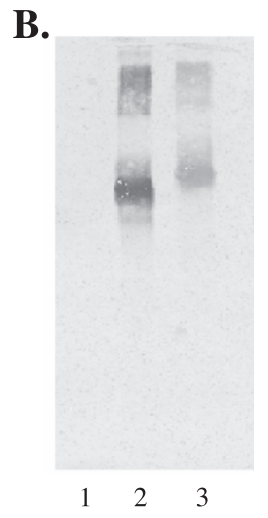

B.

C.
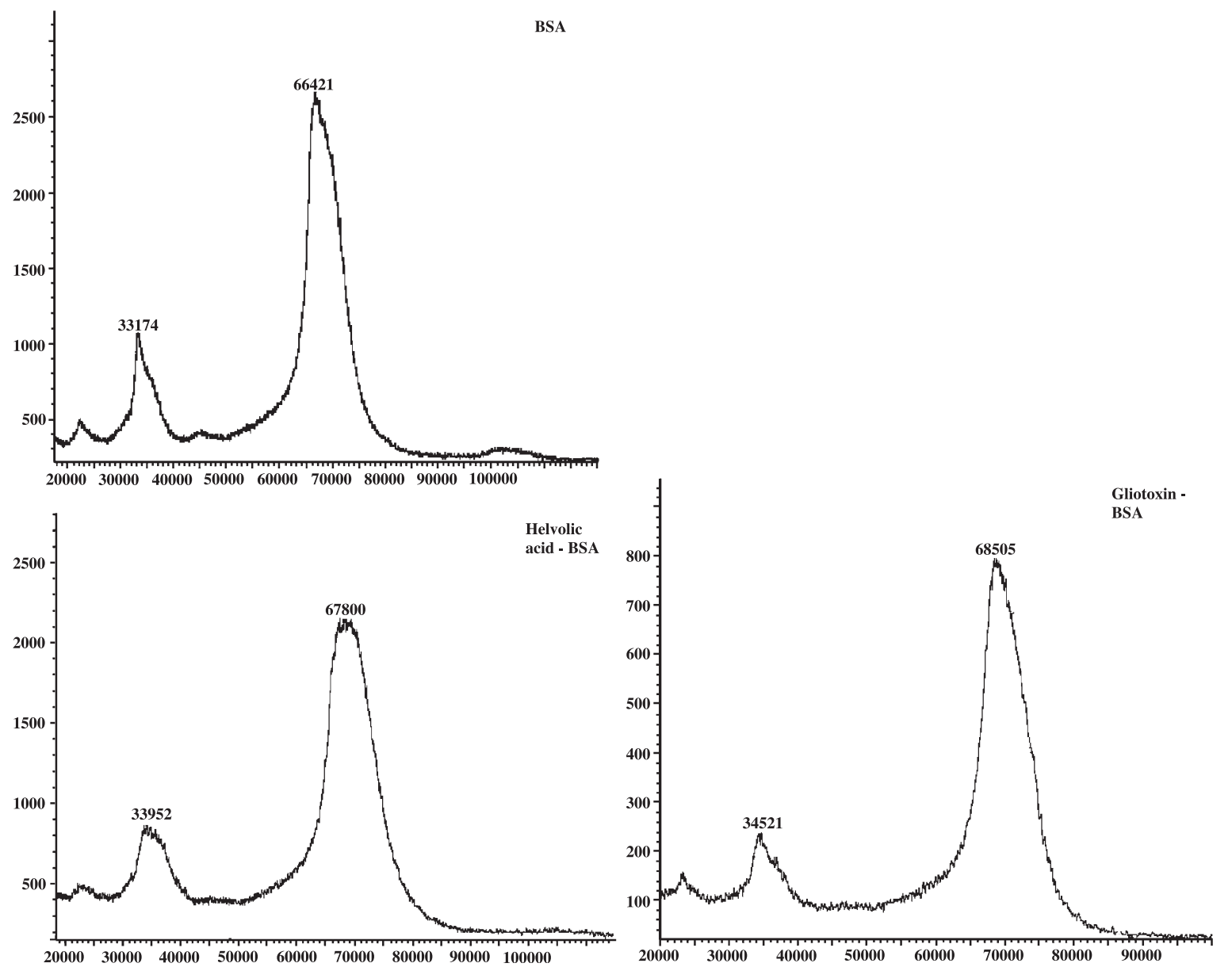

Fig. 2. (A) SDS-PAGE analysis of toxin-BSA conjugates (1 $\mu \mathrm{g} / \mathrm{lane})$. Lane 1, molecular weight markers; Lane 2, BSA; Lane 3, helvolic acidBSA and Lane 4, gliotoxin-BSA. (B) Western blot analysis of toxin-BSA conjugates using rabbit antisera (1/2000). Immunoblotting was carried out in two stages, whereby gliotoxin-BSA conjugate was initially detected when anti gliotoxin-thyroglobulin antisera was added to the nitrocellulose membrane. Subsequent membrane incubation with anti helvolic acid-thyroglobulin antisera revealed the presence of helvolic acid-BSA conjugate. Lane 1, BSA; Lane 2, Helvolic acid-BSA and Lane 3, gliotoxin-BSA. (C) MALDI-TOF mass spectrometry analysis of BSA, gliotoxin-BSA and helvolic acid-BSA conjugates. 
$(1 / 2000)$ in PBST/1\%(w/v) milk powder (Buffer B). Signal revelation was by goat $\mathrm{IgG}$ [anti-rabbit $\mathrm{IgG}$ ]HRP conjugate and the Diaminobenzidine $/ \mathrm{H}_{2} \mathrm{O}_{2}$ substrate system. Mass spectrometry was carried out using a Bruker Biflex 1V MALDI-TOF Mass Spectrometer. All samples were freeze-dried to $1 \mathrm{mg} / \mathrm{ml}$, deposited $(1 \mu \mathrm{l})$ with $1 \mu \mathrm{l}$ sinnipinic acid matrix onto a mass spectrometry slide and allowed to dry prior to analysis.

\subsection{ELISA protocols}

Toxin-BSA conjugates were individually diluted in $200 \mathrm{mM}$ sodium carbonate $\mathrm{pH}$ 9.6. Flat-bottomed microtiter plates (MaxiSorp ${ }^{\mathrm{TM}}$ Nunc-Immuno ${ }^{\mathrm{TM}}$ Mod- ules; Nalge NUNC International, Roskilde, Denmark) were subsequently coated at $37{ }^{\circ} \mathrm{C}$ for $1 \mathrm{~h}$ with saturating amounts of helvolic acid-BSA conjugates $(1 \mu \mathrm{g} / \mathrm{ml} ; 100 \mu \mathrm{l} / \mathrm{well})$. Optimal gliotoxin-BSA coating was found to be $5 \mu \mathrm{g} / \mathrm{ml}$ (100 $\mu \mathrm{l} /$ well). After coating, microtiter plates were washed twice with phosphate buffered saline $-0.05 \%(\mathrm{v} / \mathrm{v})$ Tween-20 (PBST) followed by addition of blocking solution $(1 \%(\mathrm{w} / \mathrm{v}) \mathrm{BSA}$ in coating buffer; $200 \mu \mathrm{l} / \mathrm{well})$ to stabilise bound toxin-protein conjugates and minimise non-specific binding. Immune antisera, diluted from $1 / 5000-1 / 40000$ in PBST, was added without the relevant toxin (final volume: $100 \mu \mathrm{l} /$ well). In the case of competitive ELISA formats for both toxins, microplate coating at $5 \mu \mathrm{g} / \mathrm{ml}$ was used for gliotoxin
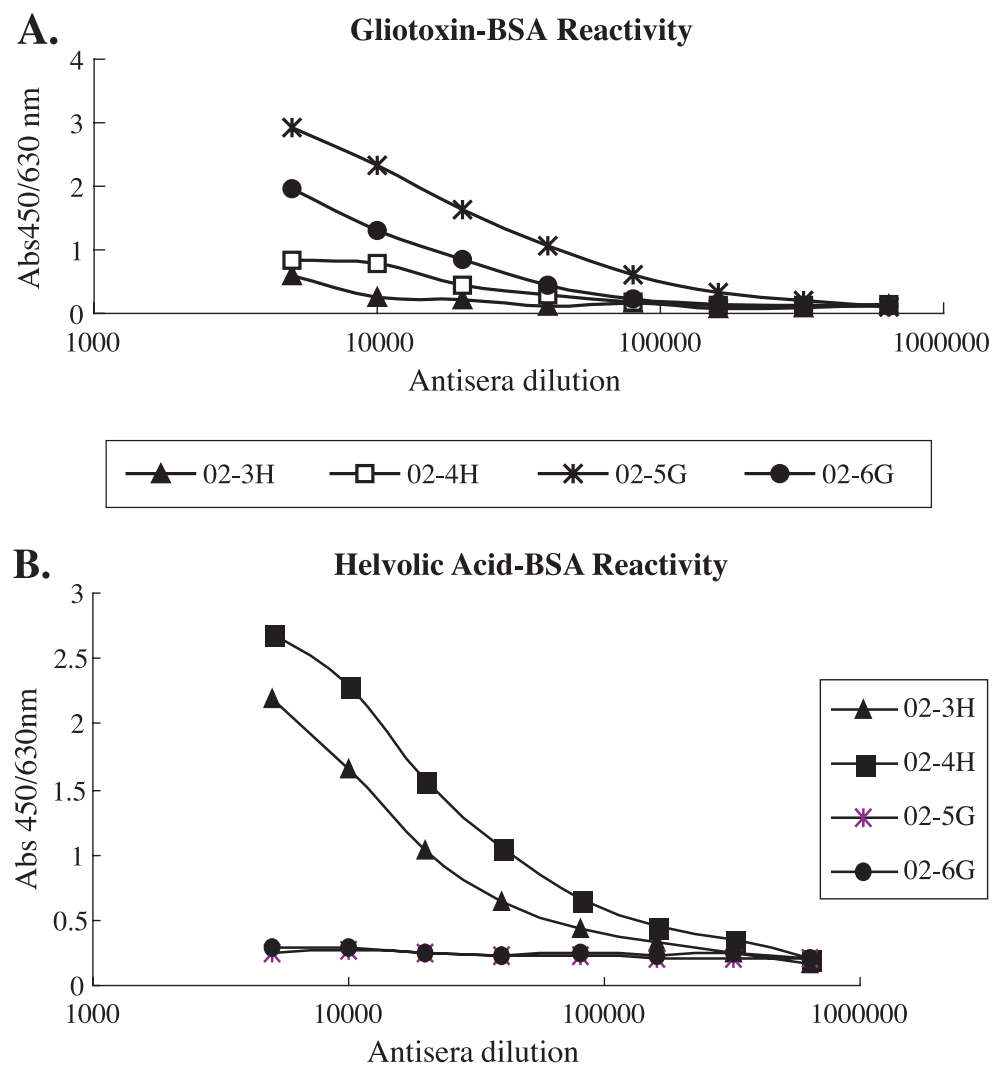

Fig. 3. Determination of antisera reactivity against toxin-BSA conjugates by ELISA. Four rabbits were immunised in total, two with each toxin-thyroglobulin conjugate. (A) Antisera reactivity against immobilised gliotoxin-BSA (02-5G and 02-6G) and (B) antisera reactivity against immobilised helvolic acid-BSA $(02-3 \mathrm{H}$ and $02-4 \mathrm{H})$. Gliotoxin-thyroglobulin antisera reveals relatively high specificity for immobilised gliotoxin BSA. Helvolic acid-thyroglobulin antisera did not bind to immobilised gliotoxin-BSA. Duplicate analysis was carried out in all cases. 
and $0.05 \mu \mathrm{g} / \mathrm{ml}$ for helvolic acid detection, respectively. In addition, relevant antisera and toxin were either added immediately or pre-incubated for 1 or $16 \mathrm{~h}$ prior to addition to the microtiter plate. The free toxin concentration range was $0-50 \mu \mathrm{g} / \mathrm{ml}$. Following incubation $(1 \mathrm{~h})$, the plate was washed four times with PBST and goat IgG [anti-rabbit IgG]-HRP conjugate added (100 $\mu \mathrm{l} /$ well; $1 / 1000$ in Buffer B) for $1 \mathrm{~h}$. The plate was washed four times and tetramethylbenzidine substrate (BioFX Laboratories, MD, USA) was added for $10 \mathrm{~min}$. The reaction was stopped by adding $1 \mathrm{~N}$ $\mathrm{H}_{2} \mathrm{SO}_{4}$ and the absorbance was read at $450 / 630 \mathrm{~nm}$ using an MRX microtitre plate reader (Dynex Technologies, West Sussex, UK).

\section{Results}

\subsection{Toxin-protein conjugate formation}

Toxin-protein conjugates were synthesised (i) to enhance the immune response against the toxin moieties and (ii) to facilitate toxin immobilisation on microplates for enzyme immunoassay. Gliotoxin- and helvolic acid-BSA conjugates were each analysed by SDS-PAGE, Western blot and MALDI-TOF mass spectrometry (Fig. 2A-C). Western blot analysis (Fig. 2B) was carried out in two stages and only gliotoxin-BSA conjugate (lane 3, Fig. 2B) was detected when anti gliotoxin-thyroglobulin antisera was added to the nitrocellulose membrane and detected as described in Section 2.5. Subsequent membrane incubation with anti helvolic acid-thyroglobulin antisera revealed the presence of helvolic acid-BSA conjugate. Unconjugated BSA was not detected by any antiserum used and high molecular weight conjugate formation is evident in Fig. 2B (lanes $2-3)$, possibly as a result of protein cross-linking. SDS-PAGE and Western blot analysis of gliotoxinBSA (Fig. 2A (lane 4) and B) resulted in the detection of a band at an apparent $\mathrm{Mr}$ of $78 \mathrm{kDa}$, which confirms a loading of $15 \mathrm{~mol}$ gliotoxin/mol BSA. Interestingly, MALDI-TOF analysis of gliotoxin-BSA indicates a hapten loading of only $4 \mathrm{~mol}$ gliotoxin/mol BSA (Fig. 2C) (see Discussion). Although SDS-PAGE did not exhibit sufficient resolution to detect bound helvolic acid, MALDI-TOF analysis for the helvolic acid-BSA conjugate confirmed a hapten loading of $2.4 \mathrm{~mol}$ helvolic acid/mol BSA. Due to the relatively large size, thyroglobulin conjugates could not be analysed by mass spectrometry and the degree of hapten loading could not be determined by SDS-PAGE due to limitations in resolution and the large protein size relative to that of the haptenylated form.

\subsection{Anti-toxin antisera titer and specificity evaluation by ELISA}

Resultant antibody titers and specificity for all both immunogens is shown in Fig. 3. Fig. 3A illustrates the reactivity of antisera raised against gliotoxin- and helvolic acid-thyroglobulin conjugates, respectively, against immobilised gliotoxinBSA over a range of antisera dilutions ranging from $1 / 5000$ to $1 / 40000$. Specific detection of immobilised gliotoxin-BSA is evident at an antiserum (anti gliotoxin-thyroglobulin) dilution of $1 /$ 40000 which was in turn selected as the optimal antiserum dilution for the detection of free glio-
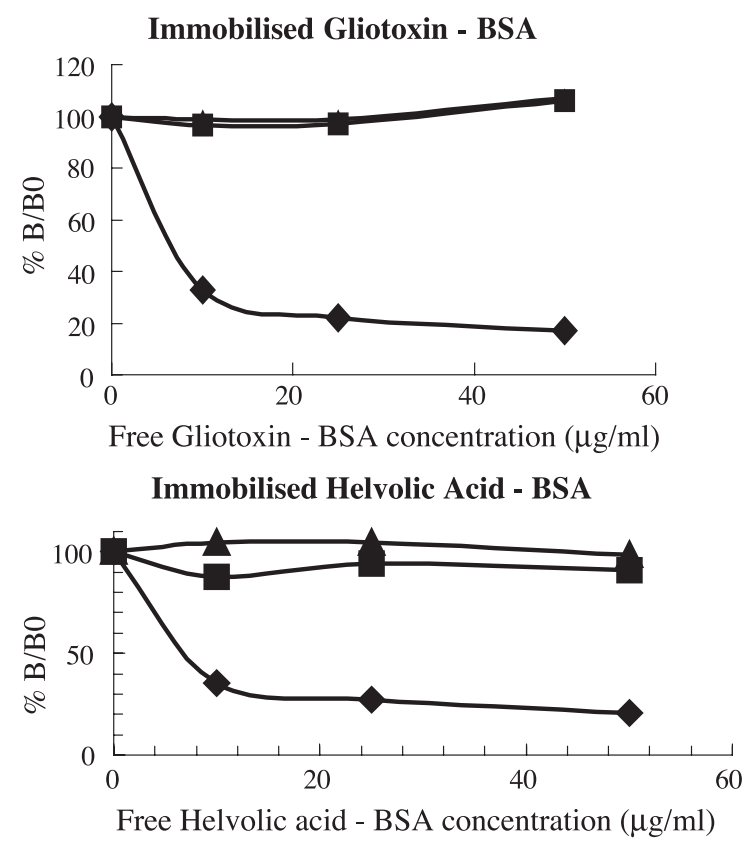

Fig. 4. Inhibition profiles for toxin-thyroglobulin antisera binding to immobilised toxin-BSA following co-incubation with cognate toxin-BSA conjugate (- - ) free BSA (- - -) or thyroglobulin (- $\mathbf{-}-)$. $\mathrm{IC}_{50}$ values ranged from 4 to $5 \mu \mathrm{g} / \mathrm{ml}$. Duplicate analysis was carried out in all cases. 
toxin. Although some reactivity is evident at lower dilutions of helvolic acid-thyroglobulin antisera, minimal cross-reactivity with bound conjugate is detected at antisera levels greater than $1 / 10000$ dilution.

The reactivity of antisera against immobilised helvolic acid-BSA conjugate is shown in Fig. 3B. Significantly, gliotoxin-thyroglobulin antisera does not bind to helvolic acid-BSA antigen at any dilution tested. In all cases, pre-immune antisera did not detect either immobilised gliotoxin- or helvolic acid-BSA conjugates. Furthermore, immune antisera did not bind to unmodified BSA immobilised at the same coating concentration as toxin-BSA conjugates, thereby confirming the specificity of the respective antisera for the conjugated toxin moieties.
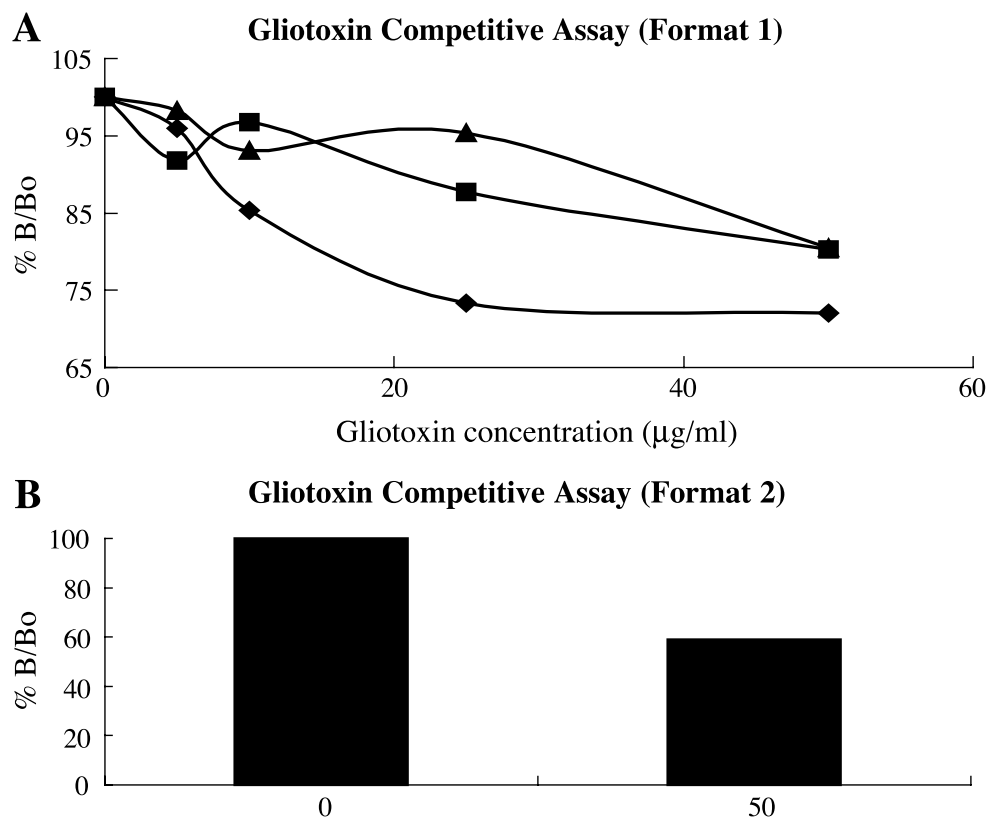

Gliotoxin concentration $(\mu \mathrm{g} / \mathrm{ml})$

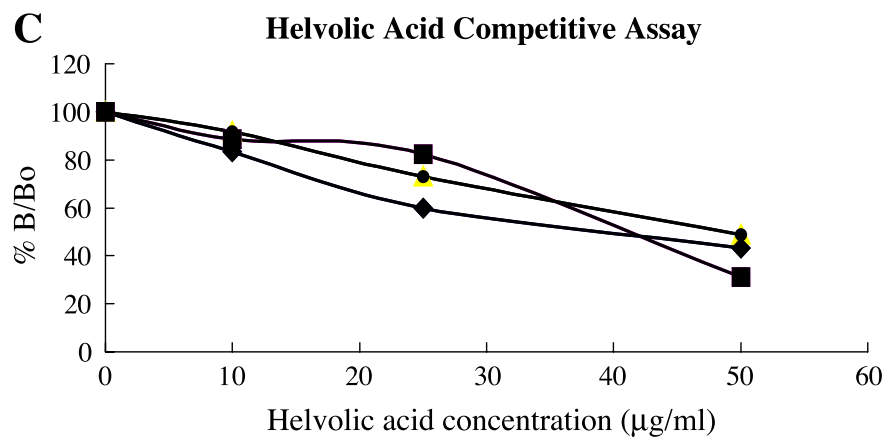

Fig. 5. (A) Inhibition of gliotoxin - thyroglobulin antisera (1/40000) binding to immobilised gliotoxin-BSA (microwell coating concentration: $5 \mu \mathrm{g} / \mathrm{ml}$ ) by free gliotoxin. Antisera and free gliotoxin were either added immediately to microwells (- $\mathbf{\Delta}-)$ or incubated for $1 \mathrm{~h}(-\mathbf{\square}-)$ or $16 \mathrm{~h}(-\boldsymbol{\nabla}-)$ prior to addition to coated microwells. (B) Inhibition (41\%) of gliotoxin-thyroglobulin antisera (1/5000) binding to immobilised gliotoxin-BSA (microwell coating concentration: $1 \mu \mathrm{g} / \mathrm{ml}$ ) by free gliotoxin. Antisera and free gliotoxin were added immediately to microwells after mixing. (C) Inhibition of helvolic acid-thyroglobulin antisera (1/2000 (- - -), 1/8000 (-ש-) or 1/32 000 (- -)) binding to immobilised helvolic acid-BSA (microwell coating concentration: $0.05 \mu \mathrm{g} / \mathrm{ml}$ ) by free helvolic acid. Antisera and free helvolic acid were added immediately to microwells and no pre-incubation was necessary. Duplicate analysis was carried out in all cases. 


\subsection{Toxin-protein conjugate inhibition of antibody binding}

Further evaluation of toxin-protein antisera was performed by determining the extent of inhibition of antisera binding due to the presence of either the appropriate toxin-BSA conjugate or free BSA. It is clear from Fig. 4 that free BSA (or thyroglobulin) does not interfere with antibody binding to the respective immobilised toxin conjugate. However, the addition of free toxin-BSA to gliotoxin- and helvolic acid-thyroglobulin antisera, respectively, at dilutions of $1 / 40000$ and $1 / 16000$, completely inhibits antibody binding to the immobilised toxins with inhibitory concentration (IC) $)_{50}$ values ranging from 4 to $5 \mu \mathrm{g} /$ $\mathrm{ml}$ in all cases.

\subsection{Inhibition of toxin-thyroglobulin antiserum binding by free toxin}

Free gliotoxin inhibits antiserum (anti gliotoxinthyroglobulin) binding to immobilised gliotoxinBSA conjugate in a time- and concentration-dependent manner. Fig. 5A shows standard curves obtained following data normalisation by $\mathrm{B} / \mathrm{Bo}$ transformation (Davies, 1994) prior to plotting against free gliotoxin concentration between 0 and $50 \mu \mathrm{g} / \mathrm{ml}$. The standard curves represent the results obtained following gliotoxin pre-incubation with antiserum (gliotoxin-thyroglobulin) for 0,1 and $16 \mathrm{~h}$ incubation prior to addition to microwells pre-coated with gliotoxin-BSA and the assay then performed as per Section 2.6. The greater degree of inhibition obtained following $16 \mathrm{~h}$ incubation of free gliotoxin with cognate antiserum suggests that relevant IgG exhibits low avidity for free gliotoxin. Microplate coating at $1 \mu \mathrm{g} / \mathrm{ml}$ gliotoxin-BSA and reduction in antiserum dilution to $1 / 5000$ (from 1/ 40000 ) facilitated a $41 \%$ reduction in antibody binding at $50 \mu \mathrm{g} / \mathrm{ml}$ gliotoxin (Fig. 5B). Apart from this, alteration in assay conditions through the use of alternative buffers/pH has not resulted in a significant improvement in assay performance. Neither has the addition of low concentrations of reducing agents such as dithiothreitol, which confirms that antibody reactivity is most likely not directed against the reduced form of gliotoxin. Fig. 5C shows a standard curve for free helvolic acid detection by competitive enzyme immunoassay as a result of the inhibition of helvolic acid-thyroglobulin antiserum binding to immobilised helvolic acid-BSA conjugate by free helvolic acid in a concentration-dependent manner. Interestingly, the time-dependent inhibition of antiserum binding to immobilised helvolic acid-BSA was not as significant as that observed for gliotoxin (data not shown). Thus, in both cases, antibodies raised against thyroglobulin-toxin conjugates were capable of recognising the free toxins in competitive enzyme immunoassay formats.

\section{Discussion}

Conventional assays for the detection of Aspergillus infection rely upon the identification of cell wall components released by the fungus during infection. While these antibody-based assays have a number of applications they can yield false positives and may also fail to detect infection where shedding of cell wall material has not occurred (Yeo and Wong, 2002). A number of protein or non-proteinaceous toxins produced by $A$. fumigatus play a crucial role in assisting the fungus to colonise and penetrate pulmonary tissue and may be detected in blood, urine or sputum specimens (Amitani et al., 1995a; Daly and Kavanagh, 2001). As a consequence, we have sought to develop a series of antibody-based immunoassays to detect fungal toxins previously implicated in tissue invasion since these could represent an improved means of identifying Aspergillosis.

The work presented here contains the first description of the preparation of thyroglobulin-based immunogens for the fungal toxins, gliotoxin and helvolic acid. We further describe the generation of sensitive and specific polyclonal antibodies, which are capable of detecting both protein-coupled, and free toxins, and finally the development of microplate-based, competitive immunoassay formats to detect both toxins.

Thyroglobulin was chosen as the carrier protein for immunisation as theoretically it should contain a greater number of amino groups available for hapten coupling relative to BSA (Hermanson, 1996). Although toxin-thyroglobulin conjugates could not be readily characterised prior to immunisation, due to their large size $(>660 \mathrm{kDa})$, the approach of simultaneous synthesis and characterisation of toxin-BSA conjugates by SDS-PAGE and MALDI-TOF mass 
spectrometry confirmed that both chemistries (EDC and PMPI/SATA) employed for protein modification were successful. Indeed, the subsequent analysis of all antisera generated confirmed the validity of this strategy.

High molecular weight conjugate formation was evident following Western blot analysis of both toxin-BSA conjugates (Fig. 2B). This is most likely due to antibody reactivity against toxin modified/EDC cross-linked BSA which was also formed during toxin-protein coupling. It is possible that similar high molecular weight conjugates were formed during toxin-thyroglobulin synthesis and may have contributed to the significant immunogenicity of resultant conjugates. Using MALDI-TOF mass spectrometry, Keough et al. (1997) have shown the maximum hapten loading of human serum albumin (HSA) to be $17.9 \mathrm{~mol}$ phthalic anhydride/mol HSA. The values for hapten coupling to BSA in the present work are somewhat lower, and possibly result from the differential reactivity of $\varepsilon$-amino groups in BSA towards activated fungal toxins relative to those in HSA. Interestingly, addition of sulpho- $N$-hydroxy succinimide to the EDC-mediated coupling reaction did not enhance conjugate formation (data not shown).

A discrepancy arose with respect to gliotoxin loading on BSA, whereby SDS-PAGE confirmed a loading of $15 \mathrm{~mol}$ gliotoxin/mol BSA while MALDITOF mass spectrometry indicated only 4 mol gliotoxin/mol BSA. Previous analysis of gliotoxin has suggested that the molecule fragments when mass spectrometry is used as a means of detection (Taylor et al., 1996), thus it is likely that gliotoxin has been cleaved or degraded from BSA conjugates resulting in the appearance of an incorrect conjugate $\mathrm{m} / \mathrm{z}$ ratio by mass spectrometry. Thus, we believe the estimate of $15 \mathrm{~mol}$ gliotoxin/mol BSA by SDS-PAGE is a more reliable estimate of hapten loading.

Initial screening of anti toxin-thyroglobulin antisera by Western blotting was supplemented by rigorous microplate enzyme immunoassay evaluation of antisera titer and specificity. Data presented in Figs. 3 and 4 confirm the specificity of two preparations of anti gliotoxin-thyroglobulin antisera $(02-5 \mathrm{G}$ and $02-$ $6 \mathrm{G})$. It is clear that antiserum $02-5 \mathrm{G}$ had a higher titer (Fig. 3) and was unreactive against immobilised helvolic acid-BSA conjugates. It was therefore used for gliotoxin detection by competitive immunoassay.
Furthermore, only gliotoxin-BSA, and neither free BSA nor thyroglobulin, was capable of inhibiting cognate antiserum binding to immobilised gliotoxin-BSA (Fig. 4). The $\mathrm{IC}_{50}$ values (Fig. 4) obtained for both toxin-protein conjugates $(4-5 \mu \mathrm{g} / \mathrm{ml})$ equates to approximately $70 \mathrm{nM}$ toxin-protein and indicates the relatively high affinity each antiserum preparation for protein coupled toxin.

Work by Chan and Ho (2002) has recently demonstrated the utility of protein-conjugated haptens in eliciting anti hapten-protein polyclonal antibodies. Here, we demonstrate that such antibodies can be further utilised to detect free, in addition to, conjugated haptens. A minimum of $15-30 \mu \mathrm{M}$ gliotoxin (5$10 \mu \mathrm{g} / \mathrm{ml}$ ) was detectable by competitive immunoassay following overnight incubation of free toxin with anti gliotoxin-thyroglobulin antiserum. This is comparable to the lower limit of sensitivity $(10 \mu \mathrm{M})$ recently reported by Tuomola et al. (2000) for the detection of 3-methylindole using anti-3-methylindole monoclonal antibodies and high sensitivity fluorometric detection. It can be seen from Fig. 5B that a $5 \times$ reduction in gliotoxin-BSA coating level (to $1 \mu \mathrm{g} / \mathrm{ml}$ ) facilitated the detection of free gliotoxin, whereby a $41 \%$ decrease in anti gliotoxin-thyroglobulin binding to immobilised gliotoxin-BSA was evident in the absence of any pre-incubation with free toxin. Initial attempts to detect free toxins using microplates coated at saturating concentrations of helvolic-BSA conjugate were unsuccessful (data not shown). Consequently, it was decided to coat microplates at lower coating concentrations of the toxin-BSA conjugate with the intent to enhance the likelihood of antibody reactivity with free as opposed to immobilised toxin. This strategy proved successful. With respect to helvolic acid, no pre-incubation with respective antiserum was required to enable the detection of free helvolic acid on microplates pre-coated at $0.05 \mu \mathrm{g} / \mathrm{ml}$ helvolicBSA conjugate (Fig. 5C). Again, a sensitivity of detection equivalent to $17 \mu \mathrm{M}$ helvolic acid $(10 \mu \mathrm{g} /$ $\mathrm{ml})$ was achieved.

In summary, hapten conjugate synthesis has facilitated the development of competitive immunoassay formats for the detection of the fungal toxins, gliotoxin and helvolic acid. Work directed towards the generation of monoclonal antibodies against these molecules, in addition to the assessment of the resultant diagnostic potential, is currently under- 
way. The ability to detect toxins secreted by Aspergillus in vivo may facilitate the rapid diagnosis of aspergillosis and result in improved treatment and recovery.

\section{Acknowledgements}

This work was funded by Enterprise Ireland Strategic Research Grant (ST/00/0078A) and, in part, by the Higher Education Authority-Programme for Research in Third Level Institutions (PRTLI): Cycle 3.

\section{References}

Amitani, R., Murayama, T., Nawada, R., Lee, W.J., Niimi, A., Suzuki, K., Tanaka, E., Kuze, F., 1995a. Aspergillus culture filtrates and sputum sols from patients with pulmonary aspergillosis cause damage to human respiratory ciliated epithelium in vitro. Eur. Respir. J. 8, 1681.

Amitani, R., Taylor, G., Eleziz, E., Jones, C.L., Mitchell, J., Kuze, F., Cole, P.J., Wilson, R., 1995b. Purification and characterisation of factors produced by Aspergillus fumigatus which affect human ciliated respiratory epithelium. Infect. Immun. 63, 3266.

Annunziato, M.E., Patel, U.S., Ranade, M., Palumbo, P.S., 1993. pMaleimidophenyl isocyanate: a novel heterobifunctional linker for hydroxyl to thiol coupling. Bioconjug. Chem. 4 (3), 212.

Belkacemi, L., Barton, R.C., Hopwood, V., Evans, E.G., 1999. Determination of optimum growth conditions for gliotoxin production by Aspergillus fumigatus and development of a novel method for gliotoxin detection. Med. Mycol. 37 (4), 227.

Chan, E.C., Ho, P.C., 2002. Preparation and characterization of immunogens for antibody production against metanephrine and normetanephrine. J. Immunol. Methods 266 (1-2), 143.

Daly, P., Kavanagh, K., 2001. Pulmonary Aspergillosis: clinical presentation, diagnosis and therapy. Br. J. Biomed. Sci. 58, 197.

Daly, P., Kavanagh, K., 2002. Immobilisation of Aspergillus fumigatus colonies in a soft agar matrix allows visualisation of A549 cell detachment and death. Med. Mycol. 40, 27.

Davies, C., 1994. In: Wild, D. (Ed.), The Immunoassay Handbook. Stockton Press, NY, USA, pp. 8-38.

Denning, D.W., 1996. Aspergillosis: diagnosis and treatment. Int. J. Antimicrob. Agents 6, 161

Denning, D.W., 1998. Invasive aspergillosis. Clin. Infect. Dis. 26, 781.

Duncan, R.J., Weston, P.D., Wrigglesworth, R., 1983. A new re- agent which may be used to introduce sulfhydryl groups into proteins, and its use in the preparation of conjugates for immunoassay. Anal. Biochem. 132 (1), 68.

Ennis, O., Corcoran, A., Kavanagh, K., Mahon, B.P., Doyle, S., 2001. Baculovirus expression of parvovirus B19 (B19V) NS1: utility in confirming recent infection. J. Clin. Virol. 22, 55.

Fraser, R.S., 1993. Pulmonary aspergillosis: pathologic and pathogenetic features. Pathol. Annu. 28, 231.

Hermanson, G.T., 1996. Bioconjugate Techniques. Academic Press, San Diego, CA, USA.

Keough, T., Lacey, M.P., Trakshel, G.M., Asquith, T.N., 1997. The use of MALDI mass spectrometry to characterise synthetic protein conjugates. Int. J. Mass Spectrom. Ion Process. 169/170, 201.

Meunier, F., 1996. Current clinical issues on mycoses in neutropenic patients. Int. J. Antimicrob. Agents 6, 135.

Mitchell, C.G., Slight, J., Donaldson, K., 1997. Diffusible component from the spore surface of the fungus Aspergillus fumigatus which inhibits the macrophage oxidative burst is distinct from gliotoxin and other hyphal toxins. Thorax 52 (9), 796.

Richardson, M.D., Kokki, M.H., 1998. Diagnosis and prevention of fungal infection in the immunocompromized patient. Blood Rev. 12, 241.

Rinaldi, M.G., 1983. Invasive aspergillosis. Rev. Infect. Dis. 5, 1061.

Taylor, G.W., Donnelly, L.E., Murray, S., Rendell, N.B., 1996. Excursions in biomedical mass spectrometry. Br. J. Clin. Pharmacol. 42 (1), 119.

Tuomola, M., Harpio, R., Mikola, H., Knuuttila, P., Lindstrom, M., Mukkala, V.M., Matikainen, M.T., Lovgren, T., 2000. Production and characterisation of monoclonal antibodies against a very small hapten, 3-methylindole. J. Immunol. Methods 240 $(1-2), 111$.

Weig, M., Frosch, M., Tintelnot, K., Haas, A., Linsmeier, B., Heeseman, J., 2001a. Use of recombinant mitogillin for improved serodiagnosis of Aspergillus fumigatus-associated diseases. J. Clin. Microbiol. 39 (5), 1721.

Weig, M., Gross, U., Domhof, S., Brunner, E., 2001b. Use of recombinant mitogillin for serodiagnosis of Aspergillus fumigatus-associated diseases. J. Clin. Microbiol. 39 (12), 4598.

Woo, P.C., Leung, A.S., Lau, S.K., Chong, K.T., Yuen, K.Y., 2001. Use of recombinant mitogillin for serodiagnosis of Aspergillus fumigatus-associated diseases. J. Clin. Microbiol. 39 (12), 4598.

Woo, P.C., Chan, C.M., Leung, A.S., Lau, S.K., Che, X.Y., Wong, S.S., Cao, L., Yuen, K.Y., 2002. Detection of cell wall galactomannoprotein Afmp1p in culture supernatants of Aspergillus fumigatus and in sera of aspergillosis patients. J. Clin. Microbiol. 40 (11), 4382.

Yeo, S.F., Wong, B., 2002. Current status of nonculture methods for diagnosis of invasive fungal infections. Clin. Microbiol. Rev. 15,465 . 